\title{
Metabolic and adaptive immune responses induced in mice infected with tissue-dwelling nematode Trichinella zimbabwensis
}

\author{
N. Onkoba ${ }^{1,2, *}$, M.J. Chimbari ${ }^{1}$, J.M. Kamau ${ }^{2,3,4}$ and S. Mukaratirwa ${ }^{4}$ \\ ${ }^{1}$ College of Health Sciences, School of Nursing and Public Health, University of KwaZulu-Natal (UKZN), Howard \\ Campus, Durban, South Africa \\ ${ }^{2}$ Tropical Infectious Diseases, Institute of Primate Research, Karen, Nairobi, Kenya \\ ${ }^{3}$ School of Medicine, Department of Biochemistry, University of Nairobi, Kenya \\ ${ }^{4}$ School of Life Sciences, University of KwaZulu-Natal, Westville Campus, Durban, South Africa
}

\begin{abstract}
Tissue-dwelling helminths are known to induce intestinal and systemic inflammation accompanied with host compensatory mechanisms to counter balance nutritional and metabolic deficiencies. The metabolic and immune responses of the host depend on parasite species and tissues affected by the parasite. This study investigated metabolic and immuno-inflammatory responses of mice infected with tissue-dwelling larvae of Trichinella zimbabwensis and explored the relationship between infection, metabolic parameters and Th1/Th17 immune responses. Sixty (60) female BALB/c mice aged between 6 to 8 weeks old were randomly assigned into $T$. zimbabwensis-infected and control groups. Levels of Th1 (interferon- $\gamma$ ) and Th17 (interleukin-17) cytokines, insulin and blood glucose were determined as well as measurements of body weight, food and water intake. Results showed that during the enteric phase of infection, insulin and IFN- $\gamma$ levels were significantly higher in the Trichinella infected group accompanied with a reduction in the trends of food intake and weight loss compared with the control group. During systemic larval migration, trends in food and water intake were significantly altered and this was attributed to compensatory feeding resulting in weight gain, reduced insulin levels and increased IL-17 levels. Larval migration also induced a Th1/Th17 derived inflammatory response. It was concluded that $T$. zimbabwensis alters metabolic parameters by instigating host compensatory feeding. Furthermore, we showed for the first time that non-encapsulated T. zimbabwensis parasite plays a role in immunomodulating host Th1/Th17 type responses during chronic infection.
\end{abstract}

Keywords: Enteric phase, Insulin, Larval migration, Th1 and Th17, Trichinella zimbabwensis.

\section{Introduction}

Trichinella zimbabwensis is a non-encapsulated zoonotic nematode that is prevalent and widely distributed in Southern Africa where it infects a wide variety of animals (Mukaratirwa and Foggin, 1999; Pozio et al., 2002; Mukaratirwa et al., 2008, 2013; La Grange et al., 2009, 2010). The parasite has potential of causing future outbreaks of human trichinellosis in subSharan Africa due to its capability to infect non-human primates and increase in risk factors like poor animal rearing systems, increased animal product movements and human-wildlife-livestock interactions (Murrell et al., 2005; Mukaratirwa et al., 2008, 2013; Gottstein et al., 2009).

Trichinella infection is acquired through ingestion of raw or undercooked meat infected with Trichinella muscle larvae (Nöckler et al., 2000). During the enteric phase of infection, the adult female worm releases up to 1500 new born larvae (NBL) in the intestines which penetrate the intestinal mucosa and migrate to the muscles (Despommier, 1998; Appleton et al., 2001; Gagliardo et al., 2002). The NBL cause temporary enteric and tissue inflammation during migration to the sites of predilection (Bruschi and Chiumiento, 2011). The migrating larvae stimulate both mucosal and systemic immune responses where the parasite utilizes host cell-biological systems to establish its parasitism (Pozio et al., 2004) without causing an overt inflammatory reaction specifically during an asthmatic reaction (Aranzamendi et al., 2013).

In the past two decades, significant studies on the immunological responses and pathological effect of encapsulating tissue-dwelling nematode, $T$. spiralis have identified that the parasite stimulates Th2 immune responses for its establishment (Maizels et al., 2004, 2009; Maizels and Yazdanbakhsh, 2008; Jackson et al., 2009). Furthermore, T. spiralis larval and adult products have also been documented to confer protection against inflammatory and auto-immune diseases in mice and humans (Finkelman et al., 2004; Khan and Collins, 2005; Del Prete et al., 2008; Wu et al., 2010; Adisakwattana et al., 2013; Aranzamendi et al., 2013; Rodgers et al., 2014). However, this has not been the case with non-encapsulated nematode like T. zimbabwensis that is endemic in Southern Africa (Pozio and Zarlenga, 2005; Pozio, 2013). This 
paucity of studies on metabolic and adaptive immune responses that are induced by $T$. zimbabwensis parasite in infected hosts has hampered efforts to improve tools for diagnosis, surveillance, management and control. In order to improve Trichinella infection surveillance and management strategies in sub-Saharan Africa, there is need to understand underlying mechanisms utilized by the parasite in metabolism and induction of immuno-inflammatory responses during chronic T. zimbabwensis infection. Thus, this study sought to determine metabolic and immuno-inflammatory responses induced in mice infected T. zimbabwensis and the relationship between infection, metabolism and Th1/Th17 type immune response.

\section{Study animals}

\section{Materials and Methods}

Sixty (60) female BALB/c mice aged between 6 to 8 weeks old were sourced from the University of Cape Town, South Africa. The mice were maintained at the Biomedical Resources Unit (BRU) of the University of KwaZulu-Natal (UKZN), Westville campus, under specific pathogen free conditions where 6 mice were placed in an individually ventilated metabolic cages (Labotec products, RSA). Mice were randomly assigned into two groups; control $(\mathrm{n}=30)$ and T. zimbabwensis infected $(\mathrm{n}=30)$ and fed daily on heat sterilized pelleted ration (Meadow feeds, RSA) and clean water was ad libitum.

\section{Ethical statement}

All experimental protocols and procedures of the study were reviewed and approved by Animals Ethics Committee of the University of KwaZuluNatal (UKZN) (ref no: 114/13/Animal) in accordance with the South African National guidelines on animal care, handling and use for biomedical research. The experiments are reported in accordance with ARRIVE guidelines (Kilkenny et al., 2013).

\section{Parasite isolation and infection of study animals}

Crocodile-derived $T$. zimbabwensis strain (Code ISS1209) larvae were obtained from whole eviscerated carcasses of stock rats that were digested as described by Kapel and Gamble (2000). At day 0, each animal in the Trichinella-infection group was infected with 500 muscle larvae (ML) through oral lavage as described by Mukaratirwa et al. (2001). At day 7, 14, 2128 and 35 post-infection (pi), six mice from each group were sacrificed, blood was collected for serum, intestines for adult worms (AW) and whole mouse carcass was digested to obtain ML.

\section{Measurement of food and water intake and body weight}

The trends in food intake per mouse were derived by weighing the food pellets (Meadow Feeds, RSA) before feeding and 24 hours after feeding the animals on daily basis. After 24 hours from dispensing the pellets to the animals the remaining pellets and pellet crumbs were collected and weighed using Boeco balance (Germany) and the difference from the initial weight was calculated as food intake.

The volume of water intake per mouse was obtained by measuring the difference between final water volume and initial water volume after 24 hours using a laboratory measuring cylinder on daily basis.

Individual mouse body weight was also measured daily using Boeco balance (Germany) and average body weights were calculated for the two groups.

\section{Blood glucose and serum insulin levels}

To determine the levels of blood glucose, whole blood was collected using a 25 gauge needle by pricking the tail vein and approximately $10 \mu \mathrm{L}$ of blood were collected on a glucometer test strip window and used to measure blood glucose using a Contour ${ }^{\circledR}$ TS glucometer according to manufacturer's instructions (Bayer, Basel, Switzerland).

To assay for the levels of insulin in serum following infection with $T$. zimbabwensis parasite, sera were collected from 6 mice from each group sacrificed at days 7, 14, 2128 and 35 pi were used to measure insulin levels using mouse insulin ELISA kit (cat. No. EZRMI13K, Millipore, Missouri, USA). Briefly, 96 well plates pre-coated with pre-titered mouse monoclonal antibodies were washed three times with $300 \mu \mathrm{L}$ of $50 \mathrm{mM}$ Tri Buffered Saline containing Tween 20. Each serum sample and a pre-determined standard were mixed with $10 \mu \mathrm{L}$ Assay buffer $(0.05 \mathrm{M}$ phosphosaline, $\mathrm{PH} 7.4$, containing $0.025 \mathrm{M}$ EDTA, $0.08 \%$ sodium azide and $1 \% \mathrm{BSA}$ ), $10 \mu \mathrm{L}$ matrix solution containing charcoal-stripped pooled mouse serum and $80 \mu \mathrm{L}$ of pre-titered biotinylated anti-insulin detection antibody per well. The plates were incubated at room temperature for 2 hours while being agitated. Plates were washed as before and $100 \mu \mathrm{L}$ of pre-titered streptavidin peroxidase conjugate solution was added per well and incubated for 30 minutes at room temperature while being agitated. The plates were washed 6 times as before and $100 \mu \mathrm{L}$ of 3,3 , , 5, 5' tetra-methylbenzidine solution added per well and placed at room temperature for 15 minutes. The reaction was stopped by adding $50 \mu \mathrm{L}$ of $0.3 \mathrm{M}$ HCL stop solution. Samples and standard optical densities were obtained by reading the plates at $450 \mathrm{~nm}$ and $590 \mathrm{~nm}$ wavelengths. A 4 parameter logistic equation was used to determine serum insulin concentrations using GraphPad PRISM version 5.04 for windows (Graph Pad Software, San Diego, CA).

\section{Measurement of cytokine concentrations}

To determine the levels of circulating Th1/Th17 cytokines in sera, mouse cytokine specific DuoSet ELISA development system kits (R\&D systems, USA) were used to measure IFN- $\gamma$ (DY485-05) and IL-17 (DY421-05) levels in serum according to manufacturer's instructions. Briefly, NUNC Maxisorp ${ }^{\circledR}$ 96-well ELISA plates were coated with either $4.0 \mu \mathrm{g} /$ 
$\mathrm{mL}$ of mouse IFN- $\gamma$ capture antibody or $2.0 \mu \mathrm{g} / \mathrm{mL}$ of mouse IL-17 capture antibody in PBS and incubated overnight at room temperature. Plates were washed three times with $300 \mu \mathrm{L}$ of PBS containing $0.05 \%$ Tween 20 and blocked with $200 \mu \mathrm{L}$ of $1 \%$ BSA in PBS and incubated for 1 hour at room temperature. Further washing was done five times and $100 \mu \mathrm{l}$ of diluted samples and standards were added to the plates. The standards used were serially diluted in incubation buffer and highest concentrations used were $2000 \mathrm{pg} /$ $\mathrm{ml}$ for IFN- $\gamma$ and $1000 \mathrm{pg} / \mathrm{ml}$ for IL-17. The plates were then incubated for 2 hours at room temperature and washed as before. Biotinylated IFN- $\gamma$ detection antibody was used at a concentration of $600 \mu \mathrm{g} / \mathrm{ml}$ and IL-17 at $400 \mathrm{ng} / \mathrm{mL}$ and incubated for 2 hours at room temperature. Plates were then washed as before and $50 \mu \mathrm{l}$ of horseradish peroxidase-conjugated streptavidin was used at a dilution of 1:2000 and incubated for 20 minutes at room temperature. The reactions were stopped by adding $50 \mu \mathrm{L}$ of $2 \mathrm{~N} \mathrm{H}_{2} \mathrm{SO}_{4}$ and absorbance obtained at $450 \mathrm{~nm}$ and $540 \mathrm{~nm}$. Cytokine concentrations were extrapolated from standards using a 4 parameter logistic equation in Graph Pad PRISM version 5.04 for windows (Graph Pad Software, San Diego, CA).

\section{Data analysis}

Cytokine, insulin, blood glucose levels, parasite loads and food and water intake measurements were expressed as means \pm standard error (SE) and analysed using repeated measure analysis of variance (ANOVA) and the levels of significance were determined by Bonferroni post-test analyses using Graph pad PRISM version 5.04 for windows (Graph pad software, San Diego, CA, USA) and a $p$-value of $<0.05$ was considered to be significant.

\section{Parasite establishment}

\section{Results}

In the intestines, we were able to recover the T. zimbabwensis AW up to 21 days post-infection (Fig. 1). Significantly more adult worms were recovered at day 7 pi compared to days to days 14 and $21 \mathrm{pi}(p<0.001)$. At days $14 \mathrm{pi}$ and $21 \mathrm{pi}$, there were no muscle larvae obtained but few larvae were recovered (average of 0.12 larvae per gram (lpg) of muscle). The number of larvae significantly increased to $16 \mathrm{lpg}$ on day $28(p<0.05)$ and peaking $(20 \mathrm{lpg})$ at day 35 $(p<0.01)$.

\section{Food and water intake}

At day 3 to $6 \mathrm{pi}$, the infected group consumed less food compared to the control group (Fig. 2). At day 7 and $18 \mathrm{pi}$, the trend in the food intake by the infected group increased exponentially in comparison with the control group $(p<0.05)$. Where at day $14 \mathrm{pi}$, the infected group consumed an average of 12 grams of food pellets per day compared to the control group which consumed an average of 4 grams of food pellets per day. At days 19

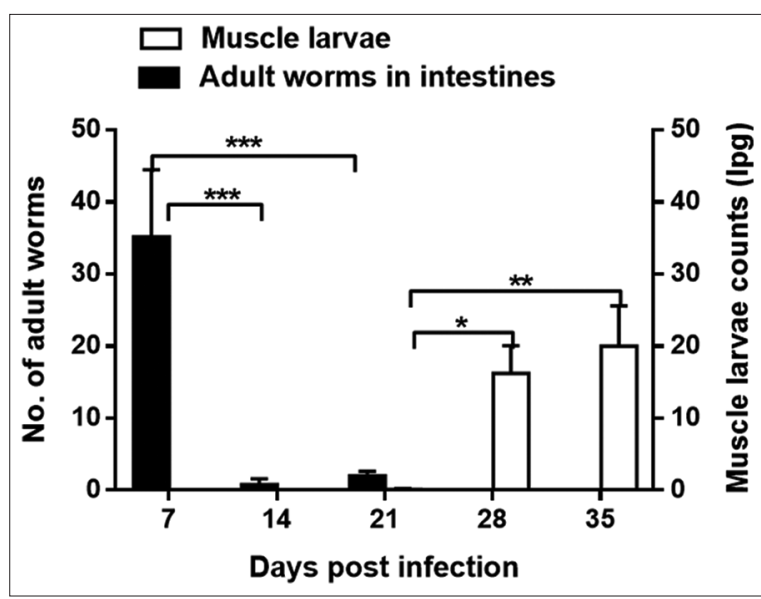

Fig. 1. Mean number of intestinal adult worms (AW) and counts of ML larvae per gram of muscle (lpg) recovered from BALB/c mice infected with Trichinella zimbabwensis. Levels of significant differences $(* P<0.05$; ** $P<0.01$, and $* * * P<0.001)$ were obtained by comparing the parasite loads in the infected and control groups.

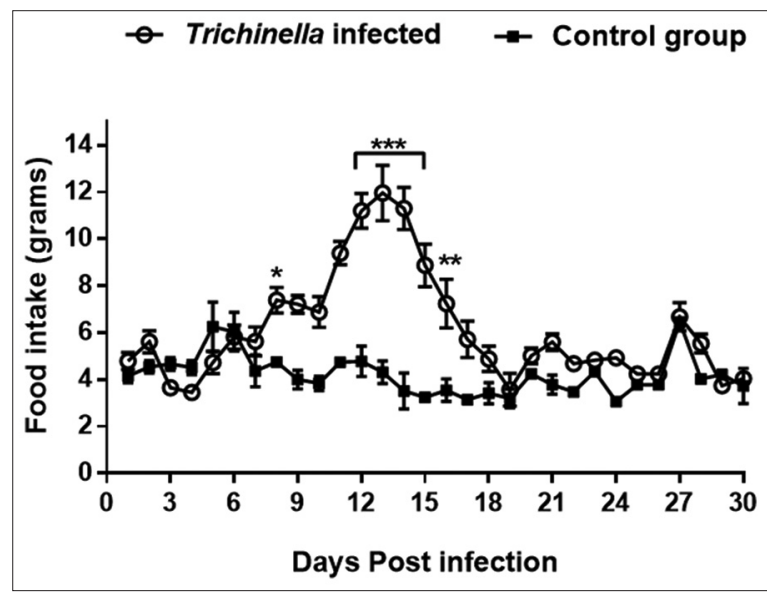

Fig. 2. Graph showing trend of food intake of mice in the infected and control groups during the experimentation period of 30 days. Levels of significant differences $(* P<0.05$; ** $P<0.01$, and *** $P<0.001)$ were obtained by comparing the infected and control groups.

to $25 \mathrm{pi}$, the trend in food intake of the infected group decreased gradually to equal that of the control group. At day 26 through $30 \mathrm{pi}$, there was no difference in the trend in food intake in both groups. Overally, the infected group had significantly higher food intake compared to the control group $(p<0.001)$.

In the two groups, a variations in the trends of water intake was observed although the infected group overally took more water than the control group. The infected group water intake at day 2 pi was higher than the control group $(p<0.01)$ (Fig. 3$)$. At days 3 to $11 \mathrm{pi}$, the water intake by the in mice in the infected group was lower than those in the control group $(p>0.05)$. 
Conversely, at days 12 to 17 pi, the control group had a higher water intake than the infected group $(p>0.05)$.

\section{Body weight}

Daily measurements of changes in body weight are shown in Fig. 4. We observed at days 3 to $11 \mathrm{pi}$, the infected group experienced significant weight loss compared to the control group $(p<0.01)$. After day $11 \mathrm{pi}$, the infected group gradually gained weight that at day 15 pi was similar to that of the control group, the upward trend further continued to peak at day 27 pi. During termination of the study (day 30), the body weight change of the infected group was greater $(p<0.01)$ than that of the control group.

\section{Insulin and blood glucose levels}

The infected group had significantly high insulin levels $(0.931 \mathrm{ng} / \mathrm{ml})$ compared to the control group $(0.302 \mathrm{ng} / \mathrm{ml})$ at day $7 \mathrm{pi}$. The insulin levels of the

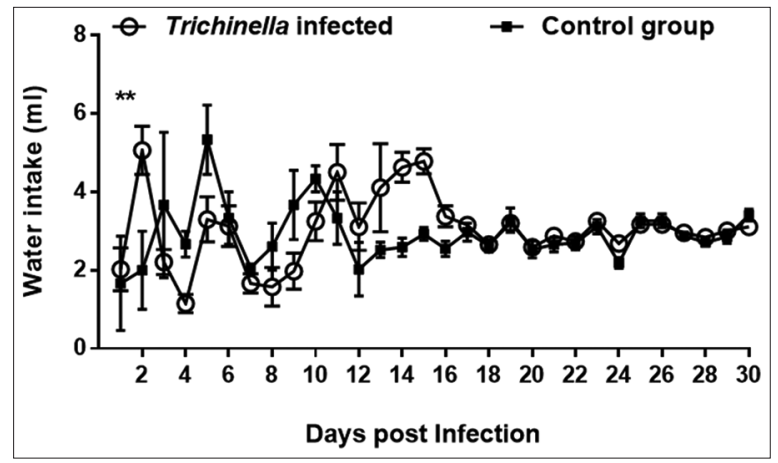

Fig. 3. Graph showing trends of water intake of mice in the infected and control groups during the experimentation period of 30 days. Levels of significant differences $(* P<0.05$; ** $P<0.01$, and $* * * P<0.001)$ were obtained by comparing the infected and control groups.

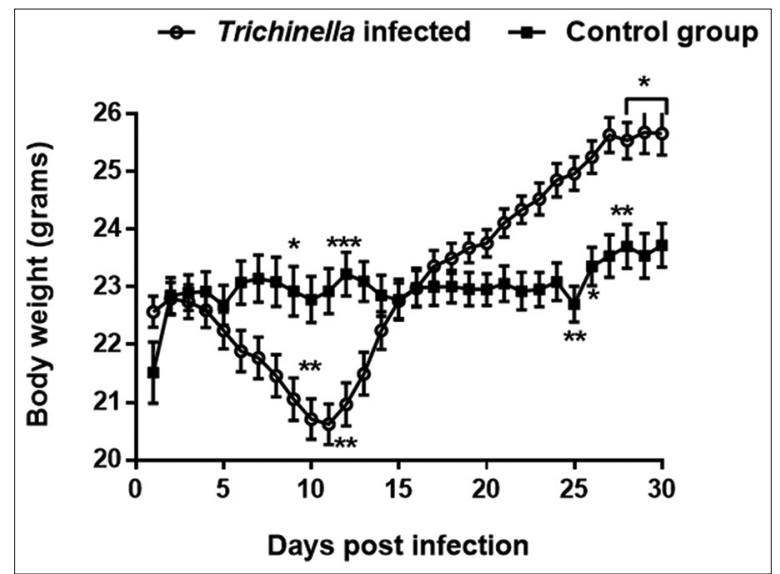

Fig. 4. Graph showing changes in mice body weight during the course of Trichinella zimbabwensis infection. Levels of significant differences $(* P<0.05 ; * * P<0.01$, and $* * * P<0.001)$ were obtained by comparing the infected and control groups. infected group dropped significantly to $0.308 \mathrm{pg} / \mathrm{ml}$ (14 dpi), $0.397 \mathrm{pg} / \mathrm{ml}$ (21 dpi) and $0.297 \mathrm{pg} / \mathrm{ml}$ (35dpi). Comparatively, the insulin levels of mice in the infected group were significantly higher $(p<0.001)$ than those in the control group (Fig. 5).

Lower blood glucose levels (101.88 mg/dL) we observed in the infected group in comparison to the control group (116.46 mg/dL). However, the differences were not significant $(p>0.05)$ (data not shown).

\section{Th1/Th17 cytokines}

The infected group had elevated levels of IFN- $\gamma$ cytokine $(404.22 \mathrm{pg} / \mathrm{ml})(p<0.001)$ at day 7 which slightly decreased at day 14pi $(295.46 \mathrm{pg} / \mathrm{ml})(p<0.05)$ and at day $21 \mathrm{pi}(118.21 \mathrm{pg} / \mathrm{ml}$ ) (Fig. 6). The IFN- $\gamma$ levels of the control group remained at baseline levels of $77.02 \mathrm{pg} / \mathrm{ml}$ throughout the experimental period.

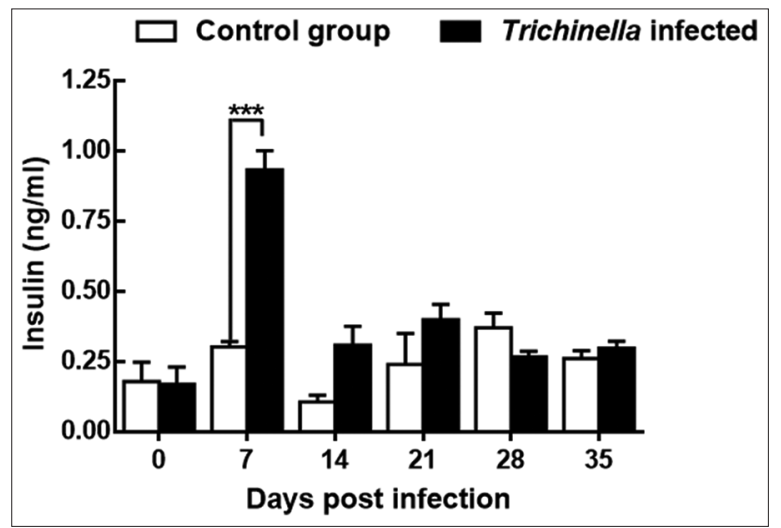

Fig. 5. Levels of insulin in mice during the course of Trichinella zimbabwensis infection. Levels of significant differences ( $* P<0.05$; ** $P<0.01$, and $* * * P<0.001$ ) were obtained by comparing the infected and control groups.

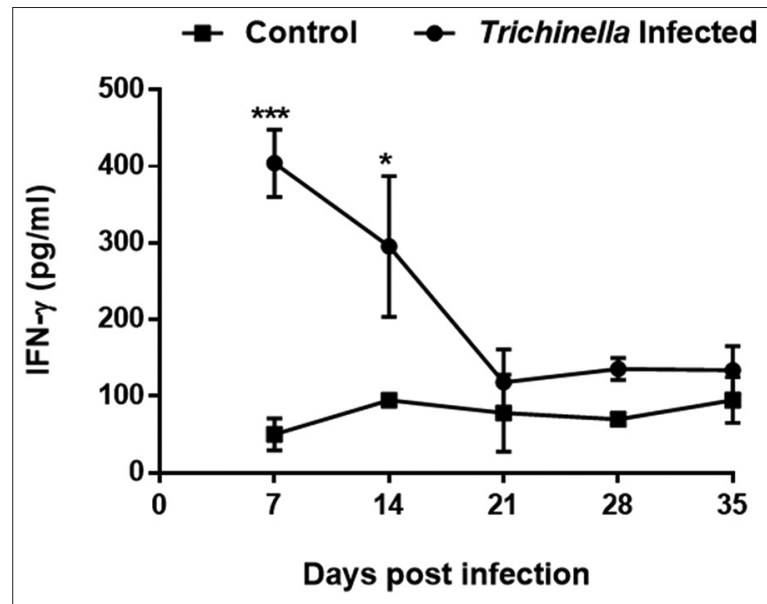

Fig. 6. Levels of Interferon gamma (IFN- $\gamma$ ) cytokine concentration during the course of Trichinella zimbabwensis infection. Levels of significant differences ( $* \quad P<0.05$; ** $P<0.01$, and $* * * P<0.001)$ were obtained by comparing the infected and control groups. 
Overall trend in IFN- $\gamma$ levels in the Trichinella infection group were significantly higher than that of the control group $(p<0.001)$. The IL-17 levels of the infected group were significantly higher $(893 \mathrm{pg} / \mathrm{ml})$ compared to the control group $(17.60 \mathrm{pg} / \mathrm{ml})(p<0.001)$ at day $14 \mathrm{pi}$ (Fig. 7).

\section{Discussion}

The results showed that $T$. zimbabwensis adult worms persisted in the small intestines for up to 21 days postinfection and few numbers of $T$. zimbabwensis ML were recovered at day 21 . This suggests that the bulk of the larval migrations is mainly at or after day 21 pi. Also an indication that ML start to arrive at their site of predilection. In addition, we established that during the enteric phase of T. zimbabwensis infection, the infected mice showed a reduced trend in food and water intake. This suggests that inflammatory reactions mediated due to NBL penetration may be playing part in hypophagic response that was accompanied with weight loss. The actual mechanism behind this phenomenon is not clear. However, it has been established by Worthington et al. (2013) that in T. spiralis-infected mice, hypophagia is due to upregulation of hormone cholecystokinin (CCK). However, the role of the intestines in limiting food ingestion, satiety and delay gastric emptying (Rigaud et al., 1994; Schonhoff et al., 2004) cannot be ruled out as factors influencing the hypophagic response observed.

We also observed that levels of blood glucose and serum insulin in Trichinella-infected mice were elevated compared with non-infected control mice. This may indicate that $T$. zimbabwensis $\mathrm{ML}$ influence glucose metabolism. However, the alteration in the metabolic responses we observed was short lived until 14 dpi when

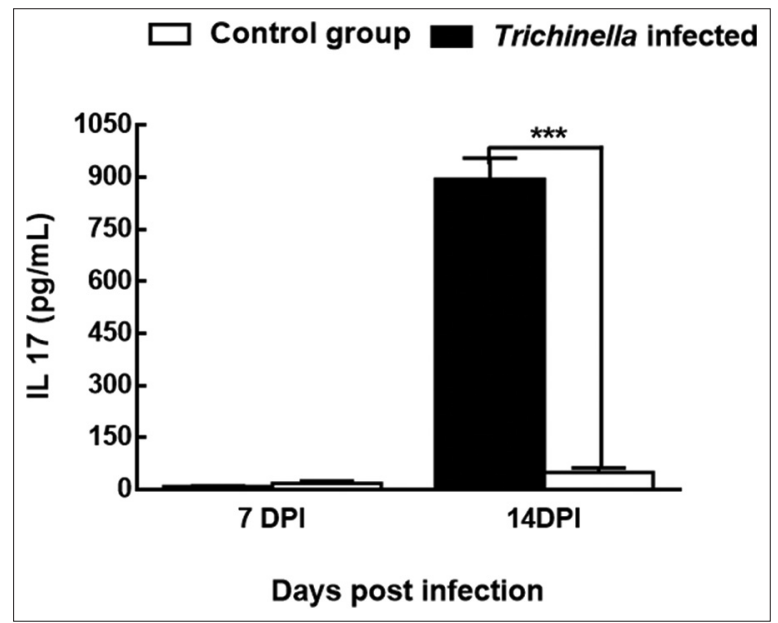

Fig. 7. Levels of Interleukin-17 (IL-17) cytokine concentration during the course of Trichinella zimbabwensis infection. Levels of significant differences $(* P<0.05$; ** $P<0.01$, and *** $P<0.001)$ were obtained by comparing the infected and control groups. the levels of insulin returned to normal suggesting that as enteritis resolved and pancreatobiliary secretomotor functions were restored. The migrating larvae may be favouring muscle glucose uptake by inhibiting glucose metabolism, glycogenolysis and controlling feeding behavior and energy expenditure (Szanto and Kahn, 2000). This may be a probable cause of fat mass accumulation and eventual body weight gain. In literature, it has been documented that accumulated fat mass deposits influence secretion of TGF- $\beta$, TNF- $\alpha$ and IL-6 cytokines that favour parasite establishment (Trayhurn and Beattie, 2001; Nehete et al., 2014) and IL-17 differentiation (Bettelli et al., 2006; Pappu et al., 2011). In the present study, we observed that there were elevated levels of pro-inflammatory cytokines, IFN- $\gamma$ and IL-17 which confirms that the known tissue-mediated innate immunity against adult T. zimbabwensis infection is initiated and inflammation is due to penetration. After $14 \mathrm{dpi}$, the levels of IFN- $\gamma$ were declining and IL-17 were undetectable showing that Th2 immune polarization limit pro-inflammatory cytokine secretion thus worm expulsion (Wakelin et al., 1994; Wu et al., 2010). This is in agreement with our previous study where we observed a decline in the levels of TNF- $\alpha$ and elevated levels of anti-Trichinellaspecific antibodies (IgG1) and Th2 cytokine (IL-4) and T-regulatory cytokine (IL-10) (Onkoba et al., 2015). This may imply that the parasite may be immuno-modulating host immune system to initiate Th2 polarisation that down regulate production of proinflammatory cytokines (Harnett, 2014).

However, the present study could not provide extensive evidence on actual mechanisms involved in glucose metabolism, Th17 secretion and regulation during $T$. zimbabwensis infection. Therefore, further studies are to be undertaken taking into consideration that $T$. zimbabwensis NBL migrates through various body cavities and tissues with diverse immunological responses that are short-lived. Immunological compartmentalization complicates the understanding of tissue-dwelling host-parasite interactions during infection.

In conclusion, T. zimbabwensis infection alters host feeding behavior similar to T. spiralis. Furthermore, we show for the first time that non-encapsulated T. zimbabwensis parasite induces Th1/Th17 type immune response during chronic infection. The data generated contributes to new knowledge on the understanding of how tissue-dwelling helminths manipulate and adapt to host metabolic and immune systems to establish parasitism. Further studies are needed to determine immune responses evoked during T. zimbabwensis NBL migratory patterns.

\section{Acknowledgements}

Our outmost gratitude goes to Mr. David Buti, Dr. Linda Bester and Dr. Sanil Singh for providing care 
and housing for study animals at BRU.

\section{Financial support}

This study received financial support from the College of Health Sciences of the University of KwaZuluNatal through PhD studentship bursary awarded to Onkoba W.N.

\section{Conflict of interest}

The authors declare that there is no conflict of interest.

References

Adisakwattana, P., Nuamtanong, S., Kusolsuk, T., Chairoj, M., Yenchitsomanas, P.T. and Chaisri, U. 2013. Non-encapsulated Trichinella spp., T. papuae, diminishes severity of DSS-induced colitis in mice. Asian Pac. J. Allergy Immunol. 31, 106-114.

Appleton, J.A., Kennedy, M. and Harnett, W. 2001. New insights into the intestinal niche of Trichinella spiralis, in: Parasitic Nematodes: Molecular Biology, Biochemistry and Immunology. pp: 103-120.

Aranzamendi, C., de Bruin, A., Kuiper, R., Boog, C.J.P., van Eden, W., Rutten, V. and Pinelli, E. 2013. Protection against allergic airway inflammation during the chronic and acute phases of Trichinella spiralis infection. Clin. Exp. Allergy 43, 103-115.

Bettelli, E., Carrier, Y., Gao, W., Korn, T., Strom, T.B., Oukka, M., Weiner, H.L. and Kuchroo, V.K. 2006. Reciprocal developmental pathways for the generation of pathogenic effector TH17 and regulatory T cells. Nature 441, 235-238.

Bruschi, F. and Chiumiento, L. 2011. Trichinella inflammatory myopathy: Host or parasite strategy? Parasit. Vectors 4, 42. http://doi. org/10.1186/1756-3305-4-42.

Del Prete, G., Chiumiento, L., Amedei, A., Piazza, M., D’Elios, M.M., Codolo, G., de Bernard, M., Masetti, M., Bruschi, F., 2008. Immunosuppression of TH2 responses in Trichinella spiralis infection by Helicobacter pylori neutrophil-activating protein. J. Allergy Clin. Immunol. 122(5), 908-913. e5.

Despommier, D.D., 1998. How does Trichinella spiralis make itself at home? Parasitol. Today 14, 318-323.

Finkelman, F.D., Shea-Donohue, T., Morris, S.C., Gildea, L., Strait, R., Madden, K.B., Schopf, L. and Urban, J.F. 2004. Interleukin-4- and interleukin13-mediated host protection against intestinal nematode parasites. Immunol. Rev. 201, 139-155.

Gagliardo, L.F., McVay, C.S. and Appleton, J.A. 2002. Molting, Ecdysis, and Reproduction of Trichinella spiralis Are Supported In Vitro by Intestinal Epithelial Cells. Infect. Immun. 70, 1853-1859.

Gottstein, B., Pozio, E. and Nöckler, K. 2009. Epidemiology, diagnosis, treatment, and control of trichinellosis. Clin. Microbiol. Rev. 22, 127-145.

Harnett, W., 2014. Secretory products of helminth parasites as immunomodulators. Mol. Biochem.
Parasitol. 195(2), 130-136.

Jackson, J.A., Friberg, I.M., Little, S. and Bradley, J.E. 2009. Review series on helminths, immune modulation and the hygiene hypothesis: Immunity against helminths and immunological phenomena in modern human populations: Coevolutionary legacies?. Immunology 126, 18-27.

Kapel, C.M.O. and Gamble, H.R. 2000. Infectivity, persistence, and antibody response to domestic and sylvatic Trichinella spp. in experimentally infected pigs. Int. J. Parasitol. 30, 215-221.

Khan, W.I. and Collins, S.M. 2005. Immune-mediated alteration in gut physiology and its role in host defence in nematode infection. Parasite Immunol. 26, 319-326.

Kilkenny, C., Browne, W.J., Cuthill, I.C., Emerson, M. and Altman, D.G. 2013. Improving bioscience research reporting: The arrive guidelines for reporting animal research. Animals 4, 35-44.

La Grange, L.J., Marucci, G. and Pozio, E. 2009. Trichinella zimbabwensis in wild Nile crocodiles (Crocodylus niloticus) of South Africa. Vet. Parasitol. 161, 88-91.

La Grange, L.J., Marucci, G. and Pozio, E. 2010. Trichinella zimbabwensis in a naturally infected mammal. J. Helminthol. 84, 35-38.

Maizels, R.M., Balic, A., Gomez-Escobar, N., Nair, M., Taylor, M.D. and Allen, J.E. 2004. Helminth parasites-masters of regulation. Immunol. Rev. 201, 89-116.

Maizels, R.M., Pearce, E.J., Artis, D., Yazdanbakhsh, M. and Wynn, T.A. 2009. Regulation of pathogenesis and immunity in helminth infections. J. Exp. Med. 206, 2059-2066.

Maizels, R.M. and Yazdanbakhsh, M. 2008. T-cell regulation in helminth parasite infections: Implications for inflammatory diseases. Chem. Immunol. Allergy 94, 112-123.

Mukaratirwa, S., Dzoma, B.M., Matenga, E., Ruziwa, S.D., Sacchi, L. and Pozio, E. 2008. Experimental infections of baboons (Papio spp.) and vervet monkeys (Cercopithecus aethiops) with Trichinella zimbabwensis and successful treatment with ivermectin. Onderstepoort J. Vet. Res. 75, 173-180.

Mukaratirwa, S. and Foggin, C.M. 1999. Infectivity of Trichinella sp. isolated from Crocodylus niloticus to the indigenous Zimbabwean pig (Mukota). Int. J. Parasitol. 29, 1129-1131.

Mukaratirwa, S., La Grange, L. and Pfukenyi, D.M. 2013. Trichinella infections in animals and humans in sub-Saharan Africa: A review. Acta Trop. 125, 82-89.

Mukaratirwa, S., Magwedere, K., Matenga, E. and Foggin, C.M. 2001. Transmission studies on Trichinella species isolated from Crocodylus niloticus and efficacy of Fenbendazole and 
Levamisole against muscle L 1 stages in BALB/ mice. Onderstepoort J. Vet. Res. 68, 21-25.

Murrell, K.D., Dorny, P., Flisser, A., Nash, T. and Pawlowski, Z. 2005. WHO/FAO/OIE Guidelines for the surveillance, prevention and control of taeniosis/cysticercosis.

Nehete, P., Magden, E.R., Nehete, B., Hanley, P.W. and Abee, C.R., 2014. Obesity related alterations in plasma cytokines and metabolic hormones in chimpanzees. Int. J. Inflam. 2014, Article ID 856749. http://dx.doi.org/10.1155/2014/856749.

Nöckler, K., Pozio, E., Voigt, W.P. and Heidrich, J. 2000. Detection of Trichinella infection in food animals. Vet. Parasitol. 93, 335-350.

Onkoba, W.N., Chimbari, M.J., Kamau, J.M. and Mukaratirwa, S. 2015. Differential immune responses in mice infected with the tissue-dwelling nematode Trichinella zimbabwensis. J. Helminthol. 90(5), 547-554.

Pappu, R., Ramirez-Carrozzi, V. and Sambandam, A. 2011. The interleukin-17 cytokine family: Critical players in host defence and inflammatory diseases. Immunology 134, 8-16.

Pozio, E. 2013. The opportunistic nature of TrichinellaExploitation of new geographies and habitats. Vet. Parasitol. 194, 128-132.

Pozio, E., Foggin, C.M., Marucci, G., La Rosa, G., Sacchi, L., Corona, S., Rossi, P. and Mukaratirwa, S. 2002. Trichinella zimbabwensis n.sp. (Nematoda), a new non-encapsulated species from crocodiles (Crocodylus niloticus) in Zimbabwe also infecting mammals. Int. J. Parasitol. 32, 1787-1799.

Pozio, E., Marucci, G., Casulli, A., Sacchi, L., Mukaratirwa, S., Foggin, C.M. and La Rosa, G. 2004. Trichinella papuae and Trichinella zimbabwensis induce infection in experimentally infected varans, caimans, pythons and turtles. Parasitology 128, 333-342.

Pozio, E. and Zarlenga, D.S. 2005. Recent advances on the taxonomy, systematics and epidemiology of
Trichinella. Int. J. Parasitol. 35, 1191-1204.

Rigaud, D., Angel, L.A., Cerf, M., Carduner, M.J., Melchior, J.C., Sautier, C., René, E., Apfelbaum, M. and Mignon, M. 1994. Mechanisms of decreased food intake during weight loss in adult Crohn's disease patients without obvious malabsorption. Am. J. Clin. Nutr. 60, 775-781.

Rodgers, D.T., Pineda, M.A, McGrath, M.A, Al-Riyami, L., Harnett, W. and Harnett, M.M. 2014. Protection against collagen-induced arthritis in mice afforded by the parasitic worm product, ES-62, is associated with restoration of the levels of interleukin-10-producing B cells and reduced plasma cell infiltration of the joints. Immunology 141, 457-466.

Schonhoff, S.E., Giel-Moloney, M. and Leiter, A.B. 2004. Minireview: Development and differentiation of gut endocrine cells. Endocrinology 145, 2639-2644.

Szanto, I. and Kahn, C.R. 2000. Selective interaction between leptin and insulin signaling pathways in a hepatic cell line. Proc. Natl. Acad. Sci. U. S. A. 97, 2355-2360.

Trayhurn, P. and Beattie, J.H. 2001. Physiological role of adipose tissue: White adipose tissue as an endocrine and secretory organ. Proc. Nutr. Soc. 60, 329-339.

Wakelin, D., Goyal, P.K., Dehlawi, M.S. and Hermanek, J. 1994. Immune responses to Trichinella spiralis and T. pseudospiralis in mice. Immunology $81,475-479$.

Worthington, J.J., Samuelson, L.C., Grencis, R.K. and McLaughlin, J.T. 2013. Adaptive Immunity Alters Distinct Host Feeding Pathways during Nematode Induced Inflammation, a Novel Mechanism in Parasite Expulsion. PLoS Pathog. 9(1), e1003122. doi:10.1371/journal.ppat.1003122.

Wu, Z., Nagano, I., Asano, K. and Takahashi, Y. 2010. Infection of non-encapsulated species of Trichinella ameliorates experimental autoimmune encephalomyelitis involving suppression of Th17 and Th1 response. Parasitol. Res. 107, 1173-1188. 